\title{
Implicaciones pronósticas de los márgenes positivos de las piezas de prostatectomía radical
}

\author{
A. Ojea Calvo, A. González Piñeiro, F. Domínguez Freire, A. Alonso Rodrigo, \\ B. Rodríguez Iglesias, J. Benavente Delgado
}

\author{
Servicio de Urología. Complejo Hospitalario Universitario de Vigo. Pontevedra.
}

Actas Urol Esp 2005; 29 (7): 641-656

\begin{abstract}
RESUMEN
IMPLICACIONES PRONÓSTICAS DE LOS MÁRGENES POSITIVOS DE LAS PIEZAS DE PROSTATECTOMÍA RADICAL

Evaluar las implicaciones de los hallazgos histopatológicas de los márgenes positivos de las piezas de prostatectomía en la recidiva bioquímica.

Material y métodos: Se analiza un grupo de 290 pacientes con cáncer de próstata clínicamente localizado que fueron tratados con prostatectomía radical retropúbica. Se desecharon todos los pacientes con tratamiento hormonal neoadyuvante y ganglios positivos. La media de edad en el momento de la cirugía era de 63 años (rango 47-73). 166 (57,2\%) eran T1c y 129 (42,8\%) T2. El tiempo medio de seguimiento fue de 4 años (rango 1-12). Se definió margen positivo como la presencia de células tumorales en contacto con la superficie tintada de la pieza quirúrgica. Fueron valorados desde diferentes puntos de vista: Margen por incisión capsular (sin evidencia de extensión extraprostática)/margen por extensión extraprostática, margen romo/margen espiculado, margen $\leq 4 \mathrm{~mm} /$ margen $>4 \mathrm{~mm}$, margen único/margen multifocal. Definimos recidiva bioquímica si el PSA supera $0,20 \mathrm{ng} / \mathrm{ml}$ en 2 determinaciones consecutivas.

Resultados: El porcentaje global de márgenes positivos fue de 65/290 $(22,4 \%)$. Las posibilidades de estar libres de recidiva a los 5 años son las siguientes: Márgenes negativos $71 \%$ vs márgenes positivos $44 \%$ ( $<<0,001$ ); márgenes por incisión capsular $84 \%$ vs márgenes por extensión extraprostática $33 \%$ ( $<<0,01$ ); márgenes romos $58 \%$ vs márgenes espiculados $26 \%$ ( $<<0,01$ ); márgenes $\leq 4 \mathrm{~mm} 57 \%$ vs márgenes $>4 \mathrm{~mm} 32 \%(\mathrm{p}<0,05)$; márgenes únicos $53 \%$ vs márgenes multifocales $0 \%(\mathrm{p}<0,01)$. $\mathrm{El}$ análisis multivariante demuestra que el PSA preoperatorio, el Gleason y el estadio anatomopatológico son los mejores predictores de recidiva bioquímica.

Conclusiones: Se establecen dos grupos de márgenes positivos. Un primer grupo con alta probabilidad de recidiva bioquímica: márgenes por extensión extraprostática, márgenes espiculados, márgenes de más de 4 mm y márgenes múltiples. Un segundo grupo con pronóstico más esperanzador en cuanto a la recidiva bioquímica: márgenes por incisión capsular, márgenes romos, márgenes $\leq 4 \mathrm{~mm}$ y márgenes únicos.
\end{abstract}

Palabras clave: Cáncer de próstata. Prostatectomía radical. Márgenes positivos. Recidiva bioquímica.

\section{ABSTRACT}

PROGNOSTIC IMPLICATIONS OF POSITIVE MARGINS IN RADICAL PROSTATECTOMY SPECIMENS

To evaluate the histopathologic implication of positive margins of prostatectomy specimens in the biochemical recurrence.

Matherial and methods: The study group consisted of 290 patients with clinically localized prostate cancer who were treated by radical retropubic prostatectomy. Patients with neoadjuvant hormonal therapy and positive lymph nodes were excluded. The mean age at the time of surgery was 63 years (range 47-73); 166 (57.2\%) patients were T1c and 124 (42.8\%) T2; the average time of folow-up was of 4 years (range 1-12). Positive surgical margins were defined as the presence of cancer cells at the surface inked of prostatectomy specimens. They were classified as: Margin for capsular incision (without extraprostatic extension evidence)/ margin for extraprostatic extension, margin with smooth rounded surface/margin with irregular surface, margin $\leq 4 \mathrm{~mm} / \mathrm{margin}$ $>4 \mathrm{~mm}$, unifocal margin/multifocal margin. We define biochemical recurrence if the PSA exceeds $0.20 \mathrm{ng} / \mathrm{ml}$ in two consecutive determinations.

Results: The overall rate of positive margins was 65/290 (22.4\%). The 5-year survival free of biochemical recurrence was as follows: Negative margins $71 \%$ vs positive margins $44 \%$ ( $\mathrm{p}<0.001)$; positive margins for capsular incision $84 \%$ vs positive margins for extraprostatic extension $33 \%$ ( $<<0.01$ ); positive margins with smooth rounded surface $58 \%$ vs positive margins with irregular surface $26 \%(\mathrm{p}<0.01)$; positive margins $\leq 4 \mathrm{~mm} 57 \%$ vs positive margins $>4 \mathrm{~mm} 32 \%$ ( $<<0.05)$; unifocal margins $53 \%$ vs multifocal margins $0 \%(\mathrm{p}<0.01)$. The multivariate analysis revealed that preoperative PSA, Gleason score and pathological classification were the best predictors of biochemical recurrence.

Conclusions: Two groups are established of positive margin. The first group with high probability of biochemical recurrence: margin for extraprostatic. The second group with less probability of biochemical recurrence: margin for capsular incision, margin with smooth rounded surface, margin $\leq 4 \mathrm{~mm}$ and unifocal margin.

Key words: Prostate cancer. Radical prostatectomy. Surgical margins. Biochemical progression. 
$\mathrm{S}_{\mathrm{p}}^{\mathrm{e}}$ define margen quirúrgico positivo en la pieza de prostatectomía radical como la extensión del tumor hasta la superficie externa de la pieza resecada, que se manifiesta en el examen microscópico por el contacto de las células tumorales con la superficie de la glándula pintada por el anatomopatólogo con tinta china ${ }^{1-3}$.

La tasa media de márgenes positivos en las piezas de prostatectomía radical se sitúa en el $28 \%$, con rangos que pueden oscilar entre el $0 \%$ y el $53 \%$. Las posibilidades de que aparezcan márgenes positivos se relacionan con el estado clínico, con el volumen tumoral, con el porcentaje de cáncer en la biopsia, con el Gleason, con la cifra de PSA preoperatorio, con el procesado anatomopatológico de la pieza, con la terapia neoadyuvante, con las posibilidades para el diagnóstico precoz del cáncer o incluso con la experiencia del cirujano. Parece ser que la incidencia de márgenes positivos va disminuyendo progresivamente, porque actualmente la mayoría de los cánceres de próstata son diagnosticados e intervenidos en estadio T1c y por la mayor experiencia quirúrgica. En un análisis de Epstein en $2001^{2}$, el autor observó que entre 1982 y 1988, el 41\% de las piezas de prostatectomía radical presentaban márgenes positivos. Ese porcentaje descendía al 16\% cuando estudiaba los años 1994 y 1995. En los tumores T1c entre 1994 y 1995 la incidencia de márgenes positivos era del 8\%. En el mismo análisis, en 1999 la incidencia de márgenes positivos, si las prostatectomías las practicaba siempre el mismo urólogo, descendía al 5,8\% $\%^{1-3}$.

Los pacientes con márgenes positivos tienen mayor probabilidad de progresión bioquímica y parece ser que también tienen más probabilidades de respuesta al tratamiento adyuvante y de rescate, con radioterapia. Aunque de forma global el $50 \%$ de los pacientes con márgenes positivos presentan recidiva bioquímica a los 5 años, no todos los márgenes positivos tienen porqué seguir la misma línea de conducta. Hay márgenes genéricamente etiquetados de extensos o de pequeños, multifocales o solitarios, de borde romo o espiculado, localizados en ápex, base prostática, cara anterior o posterolateral. Por otra parte, el valor predictivo de cada margen positivo puede variar cuando están presentes otros factores de mal pronóstico, como el Gleason y el estadio anatomopatológico ${ }^{1,4-6}$.
El objetivo de este estudio es evaluar la implicación de las diferentes características histopatológicas de los márgenes quirúrgicos de las piezas de prostatectomía radical en la recidiva bioquímica del cáncer después de la prostatectomía.

\section{MATERIAL Y MÉTODOS}

\section{Pacientes}

Se analiza la evolución de 290 pacientes con cáncer de próstata en estadio clínico T1, 2NOMO, a los que se les había practicado prostatectomía radical retropúbica; $166(57,2 \%)$ pacientes se clasificaron preoperatoriamente como T1 y 124 $(42,8 \%)$ como T2. La edad media de los pacientes era de 62,82 años (rango 47-73, mediana 63, desv. tip. 6.064). Fueron intervenidos entre julio de 1992 y diciembre de 2003. Se descartan los pacientes que fueron sometidos a bloqueo androgénico previo y los que en el análisis histopatológico resultaron con ganglios positivos. La revisión se hizo en diciembre de 2004. El tiempo mínimo de seguimiento de los pacientes es de 1 año y el tiempo medio de seguimiento de 4 años (rango 1-12, mediana 3,2 años, desv.tip. 2,52).

\section{PSA preoperatorio}

El PSA medio preoperatorio era de 14,32 (rango 1-71, mediana 8, desv.tip 7.894). El PSA se determinó con el ensayo KRYPTOR ${ }^{\mathrm{R}}$ que se fundamenta en la tecnología TRACE ${ }^{\mathrm{R}}$ (Time Resolved Amplified Cryptate Emission).

\section{Clasificación clínica, anatomopatológica y márgenes}

Los tumores se reclasifican según el sistema TNM sexta edición de 2002 UICC $^{13}$. Los criterios mínimos para la clasificación clínica fueron el tacto rectal, la ecografia transrectal y el estudio peroperatorio por congelación de los ganglios regionales si el PSA preoperatorio era superior a $10 \mathrm{ng} / \mathrm{ml}$. Si el PSA era superior a $20 \mathrm{ng} / \mathrm{ml} \mathrm{se}$ completaba el estudio preoperatorio con Gammagrafia ósea. Si los ganglios en el estudio peroperatorio eran positivos se suspendía la intervención.

Las piezas de prostatectomía siempre se fijaron durante 24 horas en formol tamponado al $10 \%$; se median tres dimensiones, se pesaban y se pintaban todos los márgenes quirúrgicos con tinta china, la mitad derecha de la próstata con 
tinta negra y la mitad izquierda con tinta roja. Una vez seca, apoyando la pieza sobre su superficie posterior, se seccionaba dando cortes paralelos cada $4 \mathrm{~mm}$ en un plano trasversal, desde el ápex hasta la base. Las porciones cónicas resultantes del corte en la zona del ápex y base, de aproximadamente $1 \mathrm{~cm}$ de longitud, se cortaban cada $3 \mathrm{~mm}$ de forma perpendicular al corte previo, para poder valorar más superficie quirúrgica de dichos bordes de resección. Se realizaba inclusión total de las porciones cónicas de la base y del ápex. Se realizaba inclusión parcial, con identificación individualizada de cada cápsula, con especial muestreo de:

1. Porciones posterolaterales de ambos lados, con muestreo de plexos vasculonerviosos.

2. Zonas prostáticas sospechosas, tanto macroscópicamente como por la clínica o biopsias previas.

3. Zonas de vesícula seminales o eyaculadores.

4. Zona fibromuscular anterior, sobre todo en los casos de inicio de la neoplasia a la zona de transición.

Cada bloque de tejido procesado en parafina se cortaba en cortes de 4 micras, que se teñian con hematoxilina-eosina para el estudio macroscópico.

En el estudio macroscópico y microscópico siempre se investigaban y se describían los siguientes aspectos en relación con el tumor y la pieza:

1. Tipo histológico del tumor y clasificación TNM.

2. Gleason.

3. Focalidad tumoral, identificando el nódulo mayor.

4. Localización del tumor y extensión a estructuras vecinas.

5. Relación con la cápsula, grasa periprostática, vesículas seminales, plexos neurovasculares, eyaculadores, cuello vesical y ápex.

6. Relación del tumor con los márgenes de resección, describiendo en caso de contacto la forma de contacto (romo/espiculado), tamaño aproximado en milímetros, etiología del margen (por incisión capsular pT2a,b,c/ por extensión extraprostática pT3,4), focalidad del margen (único/múltiple). Los már- genes se clasificaron por su localización en márgenes del ápex, márgenes posterolaterales, márgenes de la base y margen anterior. El margen positivo se definió como el contacto de las células tumorales con la superficie pintada con tinta china.

7. Presencia de PIN y su localización en relación con el tumor.

8. Lesiones del resto de la próstata como hiperplasia, prostatitis, metaplasias y cambios postraumáticos.

\section{Controles postoperatorios}

En todos los pacientes se establecieron controles periódicos con determinación de PSA en sangre, el primero a las 6 semanas de la intervención y posteriormente cada 6 meses de forma indefinida. En caso de PSA superior a $0.20 \mathrm{ng} / \mathrm{ml}$ en al menos dos determinaciones se diagnosticaba al paciente de recidiva bioquímica o fracaso bioquímico.

\section{Análisis estadístico}

Para el análisis estadístico empleamos el programa informático, SPSS versión 11.0 para Windows, de Microsoft. Las variables analizadas fueron: márgenes positivos, etiología de los márgenes positivos (incisión capsular/extensión extraprostática), características morfológicas de los márgenes positivos (romo/espiculado), tamaño de los márgenes positivos (focal/extenso), focalidad de los márgenes (único/múltiple), localización de los márgenes positivos (ápex/posterolateral/ base/anterior), PSA preoperatorio, el Gleason, el estadio anatomopatológico y recidiva bioquímica. Se hizo un análisis estadístico descriptivo y un estudio estadístico analítico, con regresión logística multivariante. Se utilizó como variable dependiente la recidiva bioquímica $\mathrm{y}$ como covariables margen (positivo/negativo), PSA $(>10 / \leq 10)$, Gleason $(\geq 7 /<7)$, estadio anatomopatológico (pT3/pTO-2). Se calculó punto de corte en mm para definir margen focal y margen extenso. Se estimó la probabilidad de recidiva bioquímica y tiempo hasta la recidiva por el método de Kaplan- Meier. Comparamos variables con la prueba t de Student y el test de Chi-cuadrado. Los resultados de las curvas de Kaplan - Meier se comparan con el test de Mantel-Haenszel. 


\section{RESULTADOS}

Variables

De las 290 piezas de prostatectomía analizadas eran $92(31,7 \%)$ pT2a, $64(22,1 \%)$ pT2b, 36 $(12,4 \%)$ pT2c, $48(16,6 \%)$ pT3a, $48(16,6 \%)$ pT3b y $2(0,6 \%)$ pT4a. El Gleason se estableció en 13 $(4,4 \%)$ pacientes Gleason 3-4, $179(61,7 \%)$ pacientes Gleason 5-6 y 98 (33,7\%) pacientes en Gleason 7-10. De las 290 piezas de prostatectomía, en $65(22,4 \%)$ se detectaron márgenes positivos y en $225(77,6 \%)$ no se detectan márgenes positivos. La mayoría de márgenes positivos se localizaron a nivel del ápex; $31(47,7 \%)$ se localizaron en ápex, $5(7,6 \%)$ en ápex/base, $2(3,1 \%)$ en ápex/base/posterolaterales, $1(1,5 \%)$ en ápex/ posterolaterales, $11(16,9 \%)$ en base y $15(23,1 \%)$ en bordes posterolaterales. Por la forma de contacto, el margen fue romo en $36(55,4 \%)$ y espiculado en 29 (44,6\%). El tamaño medio de los márgenes fue de 5,26 mm (rango 1-19, mediana 4, desv.tip. 3.768). En $33(50,8 \%)$ el margen era $\leq$ $4 \mathrm{~mm}$ y en $32(4,2 \%)$ era $>$ de $4 \mathrm{~mm}$. El margen era único en $57(87,7 \%)$ y múltiple en $8(12,3 \%)$.

$\mathrm{El}$ porcentaje de recidivas bioquímicas de la serie analizada fue de 85 (29,3\%). En los pacientes con márgenes positivos se detectó un porcentaje más alto de recidivas bioquímicas que en los pacientes con márgenes negativos, con significación estadística. De los 225 pacientes con márgenes negativos recidivaron $55(24,4 \%)$ y no recidivaron $170(75,6 \%)$. De los 65 pacientes con márgenes positivos recidivaron $30(46,2 \%)$ y no recidivaron 35 (53,8\%), $\mathrm{p}=0,001$ (Tabla 1).

La etiología del contacto del margen positivo influye significativamente en el porcentaje de

Tabla 1

La recidiva bioquímica después de la prostatectomía radical en los pacientes sin márgenes positivos en la pieza quirúrgica es del $24,4 \%$ frente al $46,2 \%$ de los pacientes con márgenes positivos $(\mathrm{p}=0,001)$.

\begin{tabular}{|c|c|c|c|c|}
\hline \multicolumn{5}{|c|}{ Recidiva bioquímica } \\
\hline & Sì & No & Total & $\mathbf{p}$ \\
\hline $\begin{array}{l}\text { Margen } \\
\text { negativo }\end{array}$ & $55(24,4 \%)$ & $170(75,6 \%)$ & 225 & \\
\hline $\begin{array}{l}\text { Margen } \\
\text { positivo }\end{array}$ & $30(46,2 \%)$ & $35(53,8 \%)$ & 65 & $\mathrm{p}=0,001$ \\
\hline Total & 85 & 205 & 290 & \\
\hline
\end{tabular}

pacientes con recidiva bioquímica. De los 19 pacientes con márgenes positivos cuya etiologia había sido etiquetada de incisión capsular, pT2a,b,c, recidivaron $3(15,8 \%)$ y no recidivaron 16 $(84,2 \%)$. De los 46 pacientes con márgenes positivos cuya etiología había sido etiquetada de extensión extraprostática, pT3/4, recidivaron $27(58,7 \%)$ y no recidivaron 19 (41,3\%), $\mathrm{p}=0,02$ (Tabla 2).

La forma de contacto del margen positivo con la superficie tintada de la pieza influye significativamente en el porcentaje de pacientes con recidiva bioquímica. De los 36 pacientes cuyas piezas de prostatectomía presentaban márgenes de contacto romo recidivaron $11(30,6 \%)$ y no recidivaron 25 (69,4\%). De los 29 pacientes con márgenes espiculados recidivaron $19(65,5 \%)$ y no recidivaron 10 (34,5\%), p=0,005 (Tabla 3).

El tamaño del margen también influye de forma significativa en el porcentaje de pacientes con recidiva bioquímica. El punto de corte, para que la cantidad de margen adquiera significación estadística para la recidiva bioquímica, se estimó en este estudio en $4 \mathrm{~mm}$. De los 33 pacientes cuyas piezas de prostatectomía presentaban márgenes focales de menos de $4 \mathrm{~mm}$ recidivaron $11(33,3 \%)$ y no recidivaron $22(66,7 \%)$. De los 32 pacientes con márgenes extensos de mas de $4 \mathrm{~mm}$, recidivaron $19(59,4 \%)$ y no recidivaron $13(40,6 \%)$, $\mathrm{p}=0,035$. (Tabla 4).

No existen diferencias significativas, en cuanto a la recidiva bioquímica, en relación con la localización del margen positivo en ápex, margen posterolateral o base. Los márgenes que denominamos múltiples, porque afectan a más de un margen, tienen un significado pronóstico peor que los márgenes únicos, que afectan a un solo margen. De los 8 márgenes positivos con localización en 2 o más márgenes recidivan $7(87,5 \%)$ y no recidivan $1(12,5 \%), p=0,020$. (Tabla 5).

\section{Análisis multivariante}

En el análisis multivariante, en el que se evalúan el Gleason, el PSA, el estadio anatomopatológico de la pieza y la situación de los márgenes, la afección de los márgenes por el tumor no es una variable que prediga de forma significativa la recidiva bioquímica del cáncer de próstata después de la prostatectomía radical. El coeficiente $\mathrm{B}$ es de 0,109 sin significación estadística $(p=0,779)$. Las 
Tabla 2

La recidiva bioquímica en los pacientes con márgenes positivos está relacionada con la etiología del margen. Los pacientes con margen positivo por incisión capsular recidivan en un $15,8 \%$ frente al $58,7 \%$ de los pacientes con márgenes por extensión extraprostática del tumor $(\mathrm{p}=0,02)$.

\begin{tabular}{lcccc}
\hline \multicolumn{5}{c}{ Recidiva bioquímica } \\
& Si & No & Total & p \\
\hline Incisión capsular (pT2a/b/c) & $3(15,8 \%)$ & $16(84,2 \%)$ & 19 & \\
$\begin{array}{l}\text { Extensión extraprostática } \\
\text { (pT3/4) }\end{array}$ & $27(58,7 \%)$ & $19(41,3 \%)$ & 46 & $\mathrm{p}=0,02$ \\
Total & 35 & 30 & 65 & \\
\hline
\end{tabular}

\section{Tabla 3}

La recidiva bioquímica en los pacientes con márgenes positivos está relacionada con la forma de contacto del margen con la superficie tintada de la pieza. Los pacientes con una forma de contacto romo recidivan en el 30,3\% mientras que los pacientes con márgenes espiculados recidivan en el 65,5\% ( $p=0,005)$.

\begin{tabular}{lcccc}
\hline \multicolumn{5}{c}{ Recidiva bioquímica } \\
& Sí & No & Total & $\mathbf{p}$ \\
\hline Margen romo & $11(30,6 \%)$ & $25(69,4 \%)$ & 36 & \\
Margen espiculado & $19(65,5 \%)$ & $10(34,5 \%)$ & 29 & $\mathrm{p}=0,005$ \\
Total & 30 & 35 & 65 & \\
\hline
\end{tabular}

\section{Tabla 4}

La recidiva bioquímica en los pacientes con márgenes positivos está relacionada con longitud del margen positivo. Los pacientes con márgenes de menos de $4 \mathrm{~mm}$. recidivan en $33,3 \%$ frente al $59,4 \%$ de los pacientes con márgenes de más de $4 \mathrm{~mm}(\mathrm{p}=0,035)$.

\begin{tabular}{lcccc}
\hline \multicolumn{5}{c}{ Recidiva bioquímica } \\
\hline & Si & No & Total & p \\
\hline Margen focal $(\leq 4 \mathrm{~mm})$ & $11(33,3 \%)$ & $22(66,7 \%)$ & 33 & \\
Margen extenso $(>4 \mathrm{~mm})$ & $19(59,4 \%)$ & $13(40,6 \%)$ & 32 & $\mathrm{p}=0,035$ \\
Total & 30 & 35 & 65 & \\
\hline
\end{tabular}

\section{Tabla 5}

La recidiva bioquímica en pacientes con márgenes positivos está relacionada con la focalidad del margen. Los pacientes que tienen afectado más de un margen recidivan en el 87,5\% de los casos con diferencias significativas en relación con los que solamente tienen afectado un margen, independientemente de la localización $(p=0,020)$. No hay diferencias significativas en cuanto a la localización del margen (Ápex, base o posterolateral).

\begin{tabular}{lcccc}
\hline \multicolumn{5}{c}{ Recidiva bioquímica } \\
\hline Margen en ápex & $9(29 \%)$ & $22(71 \%)$ & 31 & Apex/Base/Poster. vs \\
Margen múltiple & $7(87,5 \%)$ & $1(12,5 \%)$ & 8 & Múltiple p=0,020 \\
Margen en base & $6(54,5 \%)$ & $7(46,7 \%)$ & 11 & Ápex vs Base vs \\
Margen posterolateral & $8(53,3 \%)$ & $5(45,5 \%)$ & 15 & Postero. p=NS \\
Total & 30 & 35 & 65 & \\
\hline
\end{tabular}

variables que más influyen en la recidiva bioquímica del cáncer de próstata después de la prostatectomía radical son el Gleason, el PSA y el estadio anatomopatológico de la pieza de prostatectomía (Tabla 6).

\section{Tiempo hasta la recidiva}

Los resultados de las curvas de Kaplan - Meier indican que hay diferencias significativas, en cuanto al tiempo hasta la recidiva, entre el grupo de pacientes con márgenes negativos y el grupo de pacientes con márgenes positivos. La probabilidad de estar libres de recidiva los pacientes con márgenes negativos a los 5 años es del $71 \%$ frente al $44 \%$ de los pacientes con márgenes positivos $(\mathrm{p}<0,001)$. También existen diferencias significativas entre los grupos de pacientes con márgenes por incisión capsular y extensión extraprostática, márgenes romos y espiculados, márgenes $\leq 4 \mathrm{~mm}$ y márgenes $>4 \mathrm{~mm}$, márgenes unifocales y márgenes multifocales. La posibilidad de estar libre de recidiva a los 5 años los pacientes con márgenes por incisión capsular es del $84 \%$ frente al 33\% de los pacientes con márgenes por extensión extraprostática $(\mathrm{p}=0,01)$. La probabilidad de estar libres de recidiva a los 5 años los pacientes con márgenes romos es del 58\% frente al $26 \%$ de los pacientes con márgenes espiculados $(\mathrm{p}<0,01)$. La probabilidad de estar libres de recidiva a los 5 años los pacientes con márgenes $\leq 4 \mathrm{~mm}$ es del $57 \%$ frente al $32 \%$ de los pacientes con márgenes $>4 \mathrm{~mm}(\mathrm{p}=0,05)$. La probabilidad de estar libre de recidiva a los 5 años los pacientes con márgenes únicos, independientemente de su localización, es del 53\% frente al $0 \%$ de los pacientes con márgenes múltiples $(\mathrm{p}=0,01)$ (Figura 1). 
Tabla 6

En el análisis multivariante la variable Margen positivo/Margen negativo tiene menos valor que el PSA, Gleason y estadio anatomopatológico, para predecir la recidiva bioquímica del cáncer de próstata.

\begin{tabular}{lccc}
\hline Variables & Coeficiente $\boldsymbol{\beta}$ & Error Estándar & $\mathbf{p}$ \\
\hline Constante & 2,523 & 0,282 & 0,0001 \\
PSA $(>10 / \leq 10)$ & $-1,387$ & 0,309 & 0,0001 \\
GLEASON $(\geq 7 /<7)$ & $-0,835$ & 0,322 & 0,010 \\
PT (pT3/pT0-2) & $-1,678$ & 0,348 & 0,0001 \\
Margen positivo/negativo & 0,109 & 0,386 & 0,779 \\
\hline
\end{tabular}

\section{COMENTARIOS}

La frecuencia de tumor en el margen quirúrgico de las piezas de prostatectomía radical es un hecho relativamente frecuente que crea en el urólogo una situación de inquietud e incertidumbre. No todos los pacientes con márgenes positivos progresan, lo que significa que no todos los márgenes son iguales ni tienen el mismo valor pronóstico. La incidencia de los márgenes positivos es variable y existen múltiples factores que pueden incidir en la presencia de márgenes positivos. El pronóstico está asociado a las características de los márgenes. ¿Pudo el urólogo prevenir los márgenes positivos?. Tampoco existen ensayos clínicos que marquen pautas claras de conducta para el tratamiento de los márgenes positivos.

Incidencia de los márgenes positivos

La incidencia global de márgenes positivos es del $28 \%{ }^{1}$. La incidencia está relacionada con el diagnostico precoz del cáncer, con el estadiaje y

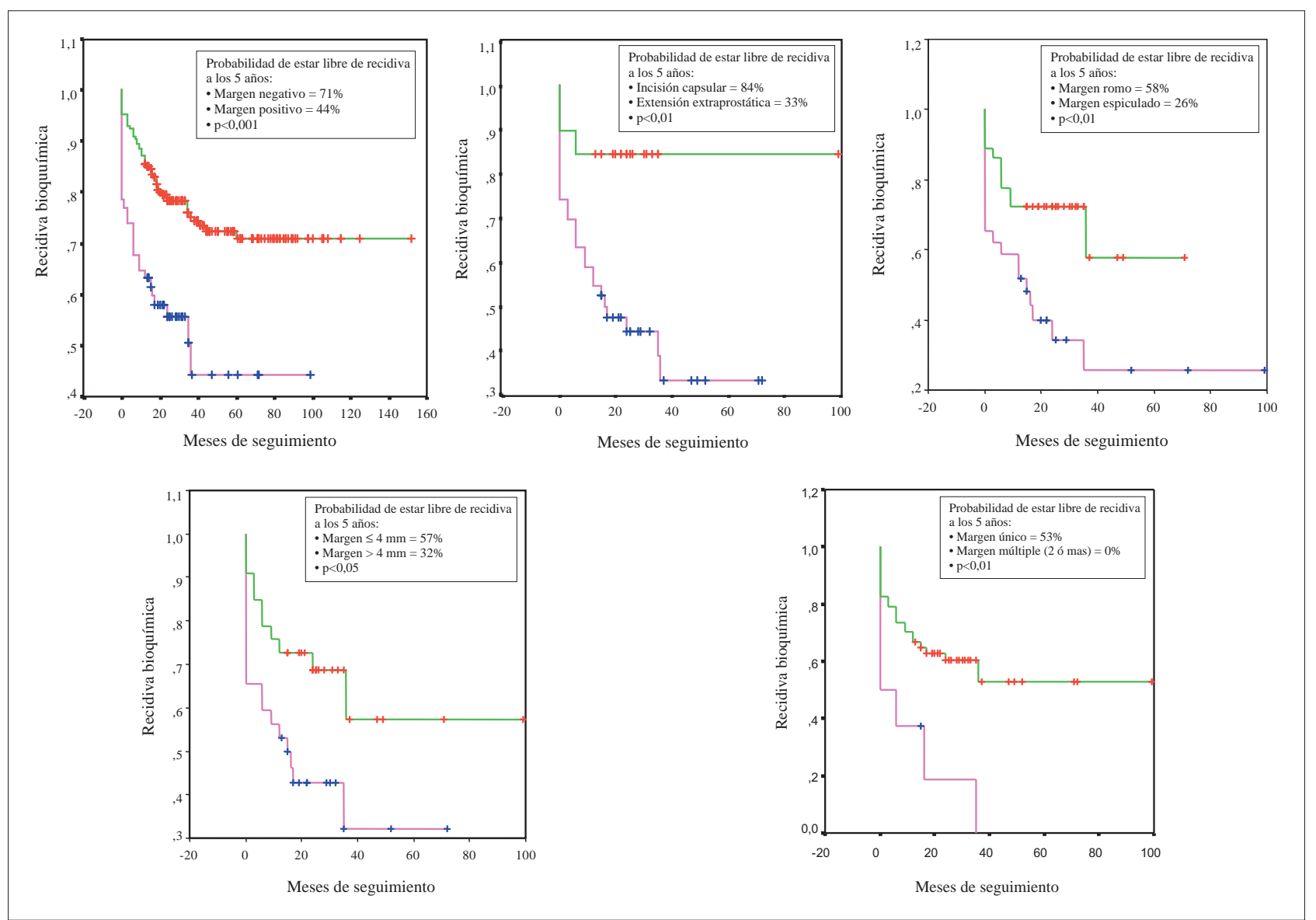

FIGURA 1. Los resultados de las curvas de Kaplan-Meier indican que hay diferencias significativas, en cuanto al tiempo hasta la recidiva bioquimica y la probabilidad de estar libres de recidiva bioquimica, entre los diferentes grupos de pacientes analizados: Pacientes con margen negativo $71 \%$ vs margen positivo $44 \%$ ( $p<0,001$ ); pacientes con margen romo $58 \%$ vs margen espiculado $26 \%$ (p<0,01); pacientes con margen $\leq 4 \mathrm{~mm} 57 \%$ vs margen $>4$ mm $32 \%$ (p<0,05); pacientes con margen unifocal $53 \%$ vs multifocal $0 \%(p<0,01)$. 
la indicación quirúrgica correcta y con una esmerada y depurada técnica quirúrgica. La incidencia global no se relaciona con la forma de abordaje quirúrgico ni con las modificaciones técnicas de preservación de la potencia o la continencia. En las prostatectomías retropúbicas se localizan fundamentalmente en el ápex, en las perineales en la base prostática y en las laparoscópicas en los márgenes posterolaterales. Cheng $\mathrm{L}$ et al. en $2000^{7}$, analizando la serie de la Clínica Mayo, observaron que el porcentaje medio de márgenes positivos era del $24 \%$. En un análisis multivariante, el nivel de PSA preoperatorio y el porcentaje de cáncer en la biopsia son factores predictivos. La combinación de los dos factores tiene un alto valor predictivo de márgenes positivos. De La Rosette JJ et al. en $2000^{8}$ revisaron el porcentaje de márgenes positivos en la prostatectomía radical laparoscópica. Los márgenes positivos oscilaban entre el 2 y el $49 \%$, comparables a los de la prostatectomía radical retropúbica. Berger AP et al. en $2002^{9}$ encontraron un porcentaje de márgenes positivos entre el $7 \%$ y el $46 \%$. El porcentaje de márgenes positivos se asociaba con el PSA preoperatorio. El porcentaje de márgenes positivos con PSA preoperatorio inferior a $4 \mathrm{ng} / \mathrm{ml}$ era pequeño. El estadio tumoral y los márgenes positivos son predictores importantes de la recidiva bioquímica. Deliveliotis $\mathrm{C}$ et al en $2002^{10}$ analizaron la relación de márgenes positivos con la preservación de los ligamentos puboprostáticos y con la preservación del cuello vesical. El porcentaje de márgenes positivos era del $21 \%$ en los pacientes en los que se le preservó el cuello vesical, del 18\% en los que se le preservó los ligamentos puboprostáticos y del $22 \%$ en los que se le preservó el cuello y los ligamentos, sin diferencias significativas en los grupos. Salomon L et al. en $2003^{11}$ evaluaron y compararon el porcentaje y la localización de los márgenes positivos en la prostatectomía radical retropúbica, perineal y laparoscópica. El porcentaje de márgenes positivos era del $18,9 \%$ en la prostatectomía retropúbica, del 13,9\% en la perineal y del 18,9\% en la laparoscópica, sin diferencias significativas, $p>0,05$. En la prostatectomía retropúbica predominaban los márgenes del ápex, en la perineal los del cuello y en la laparoscópica los posterolaterales. Guillonneau B et al. en $2003^{12}$ evaluaron los porcentajes de márgenes positivos en 1.000 prostatectomías laparoscópicas. El porcentaje de márgenes positivos era del 6,9\% en los pT2a, $18,6 \%$ en los pT2b, $30 \%$ en los pT3a y $34 \%$ en los pT3b. Las variables que mejor predecian los márgenes positivos eran el PSA preoperatorio, el estadio clínico, el estadio anatomopatológico y el Gleason. La preservación de las cintillas neurovasculares no tenía valor significativo en el riesgo de márgenes positivos ni en la recidiva bioquímica. Rassweiler $\mathrm{J}$ et al. en $2003^{13}$ compararon los posibles beneficios de la prostatectomía radical laparoscópica frente a la retropúbica en relación con los márgenes. El porcentaje de márgenes positivos no difería en los dos grupos. Era del $28,2 \%$ en la prostatectomía abierta y del $23,2 \%$ en la endoscópica. Bianco FJ et al. en $2003^{14}$ evaluaron el impacto de la preservación del cuello vesical en los márgenes positivos. La preservación del cuello vesical no incrementa el porcentaje de márgenes positivos. El porcentaje global de márgenes positivos es del 32\%: 19\% en el ápex, $2 \%$ en el cuello vesical y $11 \%$ en otras localizaciones. Cecchini Rossel L et al. en $2003^{15}$ aportaron su experiencia en prostatectomías radicales laparoscópicas. La incidencia de márgenes positivos era del $17,6 \%$. Khan MA et al. en $2003^{16}$ compararon la relación de márgenes positivos entre pacientes de menos de 50 años frente a los de más de 50 años, en la serie del Johns Hopkins Hospital de Baltimore. El porcentaje de márgenes positivos era del 3\% en los pacientes de menos de 50 años frente al 9\% en los de más de 50 años, con diferencias estadísticas, $\mathrm{p}<0,03$. Osorio Pazo F et al. en $2003^{17}$ compararon la cantidad de cáncer en la biopsia preoperatoria con el porcentaje de márgenes positivos. El porcentaje de márgenes positivos era del 14,4\% cuando estaban afectados por cáncer 20 menos de 2 cilindros y del 37,5\% cuando están afectados 4 o más de 4 cilindros. El porcentaje de márgenes positivos era del 8,7\% cuando el porcentaje de cáncer en los cilindros era inferior al $20 \%$ y del $44,1 \%$ cuando el porcentaje de cáncer era superior al $40 \%$. Brown JA et al. en $2003^{18}$ compararon las características histopatológicas de los especímenes de prostatectomía obtenidos por vía retropúbica y los obtenidos por técnica laparoscópica. El porcentaje de márgenes positivos fue 
del 20\% en las retropúbicas y del 16,9\% en las laparoscópicas, sin diferencias significativas, $\mathrm{p}>0,10$. Laven BA et al. en $2004^{19}$ analizaron la repercusión que podría implicar una pequeña modificación de la técnica quirúrgica en la disección apical para reducir la incidencia de márgenes positivos en el ápex. La modificación técnica redujo significativamente la incidencia de márgenes positivos en el ápex: la incidencia global de márgenes positivos en los tumores pT2 fue del $8,4 \%$ y la incidencia de márgenes positivos en el ápex de los tumores pT2 del 2,1\%. Serni S et al. en $2004^{20}$ evaluaron la incidencia de márgenes positivos en tumores de alto riesgo de extensión extraprostática con una modificación técnica en la prostatectomía radical anterógrada, en la que practican una amplia resección del ápex y de los elementos neurovasculares. La prostatectomía radical modificada disminuía el porcentaje de márgenes positivos en tumores de alto riesgo. El porcentaje de márgenes positivos era del 13\%. Varkarakis $J$ et al. en $2004^{21}$ evaluaron el porcentaje de márgenes positivos en pacientes de menos de 45 años de edad con cáncer de próstata. El porcentaje de márgenes positivos en pacientes jóvenes era bajo y no sobrepasaba el $7,7 \%$. Baumert $\mathrm{H}$ et al. en $2004^{22}$ analizaron la incidencia de márgenes positivos en relación con la experiencia del cirujano en la prostatectomía radical laparoscópica. $\mathrm{El}$ porcentaje de márgenes positivos era del $12,8 \%$ en los tumores pT2 y $32,8 \%$ en los tumores pT3. El número de márgenes positivos, la longitud de los márgenes y el porcentaje disminuían con la experiencia del cirujano. Ruiz L et al. en $2004^{23}$ compararon los resultados oncológicos inmediatos de dos formas de prostatectomía radical laparoscópica, transperitoneal y extraperitoneal. No encontraron diferencias significativas en cuanto a los márgenes en los dos grupos: $23 \%$ de márgenes positivos en la transperitoneal vs $29,7 \%$ en la extraperitoneal, $\mathrm{p}=0,21 ; 13 \%$ transperitoneal de márgenes positivos para los tumores pT2 vs $17 \%$ en la extraperitoneal, $\mathrm{p}=0,42 ; 43,6 \%$ de márgenes positivos para los tumores pT3 en la transperitoneal vs $44,7 \%$ en la extraperitoneal, $p=0,98$. Martorana $\mathrm{G}$ et al. en $2004^{24}$ compararon los resultados de la prostatectomía radical retropúbica frente a la laparoscópica y no encontraron diferencias signi- ficativas en cuanto al porcentaje de márgenes positivos ni en cuanto a la localización de los mismos. El porcentaje de márgenes positivos fue del $26 \%$ en la retropúbica y del $24 \%$ en la laparoscópica. Selli G et al. en $2004^{25}$ evaluaron el papel de la preservación del cuello vesical en la presencia de márgenes. El porcentaje de márgenes positivos a nivel del cuello vesical no aumentaba y mejoraba la continencia. El porcentaje global de márgenes positivos era del $22 \%$ y solamente afectaba al cuello vesical en el 5\% de los casos. Edorgru T et al. en $2004^{26}$ no encontraron diferencias significativas en cuanto a los márgenes entre la prostatectomía radical laparoscópica transperitoneal y extraperitoneal. El porcentaje de márgenes positivos fue del $22,6 \%$ en la transperitoneal y del $20,7 \%$ en la extraperitoneal. Poulakis V et al. en $2004^{27}$ predijeron el porcentaje de márgenes positivos en el 84\% de los casos, usando una red neuronal que combinaba el PSA, el Gleason y las imágenes de la resonancia. El porcentaje de márgenes positivos en su serie de prostatectomias radicales era del $22 \%$. Ward JF et al. en $2004^{28}$ observaron que la preservación de las cintillas neurovasculares no era un factor de riesgo de márgenes positivos. Curiosamente, en su experiencia, el porcentaje de márgenes positivos era menor cuando preservaron las cintillas neurovasculares que cuando no las preservaron, 34\% vs $42 \%(\mathrm{p}=0,001)$. Gaker $\mathrm{DL}$ et al. en $2004^{29}$ observaron que los márgenes positivos de las piezas de prostatectomía no estaban en relación con la preservación o no preservación de los mecanismos de la continencia a nivel de cuello vesical y ápex. El porcentaje de márgenes positivos era del 6,9\% en las que se preservaban los mecanismos de continencia y del $11 \%$ en los que no se preservaban.

\section{Etiología de los márgenes positivos}

Los márgenes en una pieza de prostatectomía radical pueden estar afectados porque el cirujano haya incidido la cápsula y no aparezca tejido periprostático en el área, porque se haya artefactado en el procesado del estudio anatomopatológico o por extensión extraprostática del tumor.

El margen anterior de la pieza de prostatectomía suele estar afectado pocas veces. La glándula a ese nivel está constituida por músculo liso, lo 
que permite al cirujano una transección del tejido con suficiente margen. Por lo tanto, la etiología de los márgenes positivos valorados a nivel del ápex siempre se considera que son secundarios a extensión extraprostática del tumor ${ }^{2,3}$. El margen basal está formado por fibras musculares del cuello vesical. El margen positivo a ese nivel, con fibras musculares de la vejiga, también se considera como secundario a extensión extraprostática del tumor ${ }^{2,3}$. Epstein ${ }^{2,3}$ asocia los márgenes positivos posterolaterales con los urólogos con menos experiencia que transeccionan la cápsula en su empeño en preservar las estructuras neurovasculares. Cuando el tumor contacta con el margen con un borde romo, liso y redondeado, lo interpreta como margen positivo por incisión capsular, por disrupción de la estrecha capa de tejido periprostático que en algún caso no sobrepasa los $0,1 \mathrm{~mm}$ de grosor. Cuando el tumor contacta con el margen quirúrgico con una forma espiculada e irregular, interpreta la etiología del margen como resección incompleta, por extensión extraprostática tumoral. Incluso cuando observa una importante reacción desmoplásica interpreta ese margen como extensión extraprostática, aunque no se vea grasa periférica ${ }^{2,3}$. El ápex es la parte de la pieza de prostatectomía que más se presta a la subjetividad del anatomopatólogo para la interpretación de las imágenes. A nivel del ápex es muy difícil determinar cuando la etiología del margen positivo se debe a incisión capsular, está artefactado por las manipulaciones o es verdaderamente positivo por extensión extraprostática del tumor. Los límites histológicos de la próstata a nivel del ápex son extremadamente vagos, sin una cápsula bien definida con glándulas entretejidas entre fibras de músculo estriado. Por otra parte, a ese nivel es en donde el cirujano libra la batalla más importante para mantener la continencia, intentado respetar la mayor cantidad posible de uretra. Epstein ${ }^{2,3}$ interpreta que el margen es secundario a incisión capsular si tumor y glándulas benignas aparecen transeccionadas en la misma área. Si no aparecen glándulas benignas en la misma área considera que la etiología del margen es por extensión extraprostática del tumor.

La etiología de los márgenes positivos también se puede asociar a la localización del margen.
Ackerman DA et al. en $1993^{30}$ analizaron la etiología de los márgenes positivos en 107 prostatectomías radicales consecutivas. A nivel del ápex, el 13\% de los márgenes positivos eran por extensión extraprostática del tumor y el $87 \%$ por incisión capsular ( $<<0,00001)$. Stamey et al. en $1990^{31}$, el $55 \%$ de los márgenes positivos a nivel del ápex se interpreta como extensión extraprostática del tumor y el $45 \%$ como incisión capsular. A nivel de los márgenes posterolaterales, el 10\% de los márgenes positivos son catalogados como extensión extraprostática y el 90\% como incisión capsular. Blute ML et al. en $1994^{32}$ situaron la incidencia más alta de márgenes por incisión capsular a nivel posterolateral.

\section{Valor pronóstico de los márgenes positivos}

Margen quirúrgico positivo no es sinónimo de tumor residual, es un factor de mal pronóstico. Aunque aproximadamente el 50\% de los pacientes con márgenes positivos presentan evidencia de progresión, no todos los márgenes positivos tienen el mismo significado. El porcentaje de recidiva bioquímica en pacientes con afectación focal es menor que en pacientes con afectación extensa. Se puede decir que los márgenes positivos resultantes de incisión capsular tienen mejor pronóstico que los márgenes positivos por extensión extraprostática; que los márgenes positivos pequeños progresan menos que los extensos. Ohori $\mathrm{M}$ et al. en $1995^{33}$ encontraron que el porcentaje de pacientes libres de recidiva era del $83 \%$ en las prostatectomías sin márgenes frente al 64\% de las que tenían márgenes positivos. Los pacientes con márgenes por incisión capsular, si el cáncer estaba localizado, se asociaban con un excelente pronóstico, que probablemente no mejoraría con terapia adyuvante. Watson RB et al. en $1996^{34}$ demostraron que las próstatas con margen positivo por incisión capsular tenían mejor pronóstico que las que presentaban márgenes por extensión extraprostática. Las primeras recidivaban en el $22 \%$ de los casos y las segundas en el 38\%. Ohori M et al. en $1999^{35}$ observaron que un alto porcentaje de márgenes positivos se presentaba a nivel del ápex, causados por incisión capsular, y que los márgenes a ese nivel no incrementan el riesgo de progresión. Cheng L et al. en $1999^{36}$ correlacionaron la etio- 
logía del margen con la supervivencia libre de recidiva bioquímica. El $78 \%$ de los pacientes con márgenes positivos por incisión capsular no recidivaba a los 5 años frente al 55\% de aquellos en los que la etiología del margen positivo era secundaria a extensión extraprostática. Zudaire Bergera JJ et al. en $1999^{37}$ estudiaron el impacto de los márgenes positivos en la recidiva biológica después de la prostatectomía radical. Concluían que los márgenes positivos tenían un alto valor predictivo de recidiva, aunque menos que el PSA mayor de $30 \mathrm{ng} / \mathrm{ml}$ y el Gleason entre 5-10. Kausik SJ et al. en $2002^{38}$ en 2002 analizaron el significado pronóstico de los márgenes positivos por extensión extraprostática, en tumores pT3a/bNOMO. El porcentaje de recidiva bioquímica en estos pacientes era del $65 \%$ si sólo tenían un margen y del $62 \%$ si tenían 2 márgenes afectos. Salomon L et al. en $2003^{39}$ analizaron las consecuencias pronósticas en relación con la localización de los márgenes en las piezas de prostatectomía. Los márgenes a nivel del ápex se asociaban a un peor pronóstico. El porcentaje libre de recidiva bioquímica a los 5 años era del $54,5 \%$ para las prostatectomías con márgenes en el ápex, del 76,9\% con márgenes en cuello vesical y del $87,9 \%$ con márgenes de localización posterolateral. Freedland SJ et al. en $2003^{40}$ no encontraron diferencias en cuanto a la recidiva bioquímica entre las prostatectomías con margen positivo por incisión capsular y las prostatectomías con extensión extraprostática del tumor, con márgenes o sin márgenes positivos. Recomiendan modificar la clasificación TNM. Pettus JA et al. en $2004^{41}$ analizaron la relación entre las características de los márgenes positivos y la localización, con la recidiva bioquímica después de la prostatectomía radical. Observaron recidiva bioquímica en $9,3 \%$ de los pacientes sin márgenes positivos. Encontraron recidiva bioquímica en el $21,4 \%$ de piezas con márgenes positivos en ápex, en el $26,3 \%$ de los márgenes de localización no apical y en el 30,8\% de los márgenes múltiples, sin diferencias significativas entre los tres grupos. En el análisis univariante todos los márgenes son predictivos de recidiva. En el análisis multivariante el margen apical no es un predictor independiente de recidiva. Connolly SS et al. en $2004^{42}$ examinaron la hipótesis de que un solo margen positivo a nivel del ápex no estaría asociado con posibilidad de incremento de recidiva bioquímica. En su análisis concluyeron que los pacientes sin márgenes recidivaban en un $7,8 \%$, los pacientes con márgenes positivos en el $42,9 \%$ $\mathrm{y}$ los pacientes con un margen positivo a nivel apical recidivaban en el $28 \%$. Por lo tanto, todos los pacientes con un margen positivo, independientemente de que se localice a nivel del ápex, presentan un riesgo más alto de recidiva bioquímica. Weldon VE et al. en $1995^{43}$ dividieron a los márgenes positivos en focales, si solamente aparecían en una sección con una longitud igual o inferior a $3 \mathrm{~mm}$, y márgenes extensos si median más de $3 \mathrm{~mm}$ o aparecían en más de un corte. Relacionan la localización y la extensión del margen con la recidiva bioquímica. Observaron que la recidiva bioquímica después de la prostatectomía radical estaba asociada a los márgenes positivos de más de $3 \mathrm{~mm}$ y a los márgenes múltiples. Fesseha $\mathrm{T}$ et al. en $1997^{44}$ no encontraron diferencias significativas en cuanto a la recidiva en las piezas de prostatectomía con márgenes positivos de menos de $8 \mathrm{~mm}$ y las de más de $8 \mathrm{~mm}$ En esos casos asociaron la recidiva bioquímica al Gleason.

\section{Prevención de los márgenes positivos}

Para minimizar los márgenes quirúrgicos positivos se considera fundamental tener: buena información en relación con el estadio, volumen y localización tumoral, antes de la cirugía y durante el acto quirúrgico; buena disposición y experiencia quirúrgica; programas de detección y diagnóstico precoz del cáncer de próstata. El tratamiento neoadyuvante antiandrogénico enmascara los resultados e induce a crear falsas expectativas. A corto plazo disminuye los márgenes positivos y a largo plazo no influye sobre la recidiva bioquímica. Shah $\mathrm{O}$ et al. en $2001^{45}$ determinaron la utilidad de la información que generaban las biopsias peroperatorias por congelación a nivel del ápex para reducir el porcentaje de márgenes positivos. Resecaban y remitían 2 ó $3 \mathrm{~mm}$ de tejido apical para estudio anatomopatológico peroperatorio y, si el margen de la biopsia por congelación era positivo, seguían resecando hasta que se negativizase. Los resultados revelaban que el $39 \%$ de los márgenes no contenían próstata, el $54 \%$ contenían glándulas prostáticas benignas y 
el 7\% cáncer de próstata. El método se mostró eficaz para reducir el tejido prostático residual después de la cirugia: sensibilidad 57\%, especificidad $86 \%$, valor predictivo positivo $25 \%$ y valor predictivo negativo 96\%. Soulie M et al. en $2001^{46}$ evaluaron las posibilidades de la resonancia magnética transrectal en tumores $\mathrm{T} 1 / 2$ para predecir márgenes quirúrgicos positivos. Los resultados demostraban que la resonancia magnética preoperatoria es una técnica adecuada para predecir el riesgo de margen quirúrgico en pacientes candidatos a prostatectomía radical con cáncer de próstata clínicamente localizado. Soloway MS et al. en $2002^{47}$ publicaron los resultados de la ablación adrogénica neoadyuvante en cánceres de próstata T2bNxM0. La deprivación androgénica disminuía los márgenes quirúrgicos positivos pero no disminuía la recidiva bioquímica a largo plazo. A los 5 años no hay diferencias en la recidiva bioquímica entre el grupo de pacientes que había recibido tratamiento antiandrogénico y el que no lo había recibido. El 64,8\% de los pacientes con tratamiento neoadyuvante están libres de recidiva y de los pacientes sin tratamiento adyuvante están libres de recidiva el 67,6\% ( $p=0,663)$. Aus G et al. en $2002^{48}$ analizaron los efectos del bloqueo androgénico neoadyuvante a corto y a largo plazo. A corto plazo disminuía el porcentaje de márgenes positivos del $45,5 \%$ al $23,6 \%$ $(\mathrm{p}=0,016)$. A largo plazo no encontraron diferencias en cuanto a la recidiva bioquímica. El porcentaje de pacientes libres de recidiva, con un seguimiento de 7 años, era del 49,8\% en los pacientes con tratamiento neoadyuvante y del $51,5 \%$ en los pacientes sin tratamiento adyuvante. Sofer M et al en $2002^{49}$ analizaron si el procedimiento quirúrgico de preservar las bandeletas neurovasculares puede influir en los márgenes positivos y en la recidiva bioquímica después de la prostatectomía. El porcentaje de márgenes positivos era del $24 \%$ con la preservación neurovascular y del 31\% sin la preservación neurovascular. El riesgo de recidiva bioquímica a los 5 años era del 14,4\% con la preservación neurovascular y del 21,1\% sin la preservación vascular, sin significación estadística. El mantenimiento de los elementos neurovasculares no influía ni en los márgenes positivos ni en la recidiva bioquímica. Goharderakhshan RZ et al. en $2002^{50}$ evaluaron la utilidad de la biopsia peroperatoria de los márgenes quirúrgicos a nivel de las bandeletas neurovasculares. Si la biopsia era positiva proseguían la resección de la cintilla neurovascular correspondiente y comparaban los resultados con los de la pieza quirúrgica. El valor predictivo positivo y negativo de las biopsias peroperatorias por congelación era respectivamente del 73\% y del 94\%. En pacientes seleccionados de alto riesgo la biopsia peroperatoria por congelación puede ser un elemento útil para conocer el estado del margen quirúrgico. Eastham JA et al. en $2003^{51}$ analizaron el papel que representa el cirujano en la presencia de márgenes positivos. Concluían diciendo que, los datos clínicos y patológicos son potentes predictores de márgenes positivos, la técnica cuidada y depurada individualmente por cada cirujano era también un factor pronóstico. La cifra de márgenes positivos mejoraba para cirujanos de gran experiencia. Por lo tanto, la experiencia y la atención cuidadosa de detalles quirúrgicos pueden reducir los porcentajes de márgenes quirúrgicos. Shah $\mathrm{O}$ et al. en $2003^{52}$ desarrollaron un algoritmo con el objetivo de disminuir el porcentaje de márgenes positivos después de la prostatectomía radical. Decidian resecar los elementos neurovasculares en relación con el Gleason, permeación perineural y porcentaje de cáncer de la biopsia. Resecaban la cintilla neurovascular en tumores con: Gleason $\leq 6$ y más del 50\% de cáncer en la biopsia preoperatoria; tumores con Gleason 7 y más del 30\% de cáncer en la biopsia preoperatoria; tumores con Gleason 8-10 y más del 10\% de cáncer en las biopsias preoperatorias o invasión perineural. Obtuvieron un descenso en la incidencia de márgenes positivos que descendió del 14\% al 8\% (p=0,027). Klotz $\mathrm{LH}$ et al. en $2003^{53}$ aportaron los resultados a largo plazo del tratamiento con Acetato de Ciproterona neoadyuvante en la prostatectomía radical frente a prostatectomía sola. El tratamiento hormonal disminuía los márgenes quirúrgicos positivos en un 50\%, sin embargo la progresión tumoral a los 6 años era la misma en los dos grupos. Fromont $\mathrm{G}$ et al. en $2003^{54}$ usaron biopsias peroperatorias por congelación para monitorizar la resección de las cintillas neuromusculares en la cirugía laparoscópica por cáncer de próstata. Con ese procedimiento reducían 
los márgenes quirúrgicos positivos del $33 \%$ al $12 \%$. Lepor $\mathrm{H}$ et al. en $2004^{55}$ determinaron el valor de la biopsia por congelación en los márgenes quirúrgicos. Las biopsias intraoperatorias del ápex contribuían a reducir los márgenes positivos en un $3,8 \%$. Prezioso D et al. en $2004^{56}$ evaluaron el tratamiento hormonal neoadyuvante con Leuprolide y Acetato de Ciproterona en la prostatectomía radical. Los márgenes positivos eran más comunes en el grupo que no había recibido tratamiento frente al que había recibido tratamiento, $60 \%$ vs. $39 \%$. No encontraron diferencias en cuanto a la facilidad de la disección, duración de la cirugía o sangrado. Hricak $\mathrm{H}$ et al. en $2004^{57}$ evaluaron el papel de la resonancia magnética endorrectal en la decisión de preservar o resecar los espacios neurovasculares durante la prostatectomía radical. La resonancia magnética alteró los planes del cirujano en el 39\% de los casos, en cuanto a la actitud sobre las cintillas neurovasculares, con información concerniente a la localización y extensión del tumor. Los datos que aportó la resonancia magnética facilitaron la decisión de preservar o resecar las cintillas neurovasculares. Ukimura $\mathrm{O}$ et al. en $2004^{58}$ describieron y analizaron los aspectos técnicos y los resultados de los ultrasonidos en tiempo real durante la prostatectomía radical laparoscópica. Los ultrasonidos transrectales en tiempo real identifican el plano entre el cuello vesical y la base prostática; identifican los elementos anatómicos del ápex para disminuir los márgenes positivos; localizan los nódulos tumorales próximos a la cápsula para que el cirujano amplíe la resección a ese nivel. Los resultados previos sugieren que la ecografia transrectal en tiempo real identifican los elementos anatómicos del área prostática y que cabe esperar beneficios oncológicos de su utilización. Han M et al. en $2004^{59}$ revisaron la incidencia de márgenes quirúrgicos positivos en la prostatectomía radical en las dos últimas décadas. Observaron que la incidencia de márgenes positivos venía descendiendo de forma global desde 1982. Al estratificar los datos, observaban que la incidencia de márgenes positivos en los tumores pT3 se mantenía constante, después de una tasa inicial del 53\% en 1982, y se estancaba en porcentajes entre el $22,7 \%$ y $27,8 \%$. De su análisis concluían que el porcentaje de márgenes positivos viene determinado por el diagnóstico precoz. El descenso en el porcentaje de márgenes positivos se debe más a la migración del estadio por el diagnóstico precoz y por la selección de pacientes que a los avances en las técnicas quirúrgicas.

\section{Tratamiento de los márgenes positivos}

Un margen quirúrgico positivo en la pieza de prostatectomía nos conduce a un escenario en el que son posibles cuatro situaciones diferentes. La primera, que exista cáncer residual limitado al lecho prostático. La segunda, que no exista cáncer residual. La tercera, que exista cáncer residual en el lecho prostático y haya metástasis microscópicas a distancia. La cuarta, que no exista cáncer en el lecho prostático y haya metástasis a distancia. Solamente en la primera de las situaciones sería posible proporcionar un beneficio al paciente con intención curativa, bien con radioterapia adyuvante, inmediatamente después de la cirugía, bien con radioterapia de rescate, retrasándola hasta que aparezca la recidiva bioquímica. Sin embargo, no existen actualmente estudios en Fase III que validen una u otra modalidad terapéutica, ni que evalúen el papel de la hormonoterapia en estos pacientes ${ }^{60-62}$. La opinión más generalizada es que los pacientes con márgenes positivos, Gleason intermedio o bajo, con PSA preoperatorio $\leq 10,9 \mathrm{ng} / \mathrm{ml}$ pueden obtener beneficio del tratamiento con radioterapia adyuvante. Los pacientes con márgenes positivos, con Gleason $<8$, PSA postoperatorio $<2 \mathrm{ng} / \mathrm{ml}$ y tiempo de duplicación de PSA superior a 10 meses, son candidatos a sacar beneficio de la radioterapia de rescate. La hormonoterapia adyuvante puede ser útil en aquellos pacientes con cánceres indiferenciados, con Gleason $>7$. Gossfeld GD et al. en $2000^{63}$ crearon un modelo de decisiones para la ayuda en el manejo de los pacientes con márgenes positivos después de la prostatectomía radical. El modelo matemático, creado por un grupo de expertos basándose en la experiencia y en la literatura, recomendaba radioterapia adyuvante para los pacientes con múltiples márgenes positivos, con Gleason bajo o intermedio y sin evidencia de invasión de las vesículas seminales. Han $\mathrm{M}$ et al. en $2001^{64}$ determinaron cuando el fracaso bioquímico en 
los pacientes con Gleason 7 y márgenes positivos podría deberse a enfermedad metastásica o recidiva local, que a su vez pudiese ser eliminada con radioterapia adyuvante. La conclusión fue que la recidiva bioquímica de estos pacientes raramente se debe a recidiva local. Por lo tanto, la radioterapia adyuvante no es eficaz en pacientes con Gleason alto y márgenes positivos, recomendando buscar nuevas pautas de tratamiento sistémico. Anscher MS en $2001^{65}$ recomendaban radioterapia adyuvante en los pacientes con alto riego de recidiva local y bajo riesgo de metástasis a distancia: pacientes con PSA indetectable después de la cirugía y márgenes positivos. Do LV et al. en $2002^{66}$ trataron con radioterapia adyuvante o de rescate a dos grupos de pacientes con signos histopatológicos adversos, márgenes positivos, extensión extracapsular e invasión de las vesículas seminales. Concluyeron diciendo que ni la radioterapia adyuvante ni la radioterapia de rescate eran eficaces cuando a los criterios anteriormente descritos se asociaban otros factores adversos, como Gleason 8-10 y PSA preoperatorio mayor de $20 \mathrm{ng} / \mathrm{ml}$. Hachiya $\mathrm{T}$ et al. en $2002^{67}$ comparan la hormonoterapia adyuvante y observación en pacientes con márgenes positivos después de la prostatectomía radical. El porcentaje de pacientes libres de recidiva bioquímica a los 5 años fue del 85,9\% en el grupo de hormonoterapia y del $80 \%$ en el grupo de observación, sin diferencias significativas. En este estudio, haciendo estratificaciones, parece que pudiesen obtener beneficio los pacientes con los tumores más indiferenciados. Lennernas B et al. en $2003^{68}$ observaron que la radioterapia adyuvante puede incrementar el control local en tumores pT3/4 con afectación de vesículas seminales y/o márgenes positivos $\mathrm{y} / \mathrm{o}$ Gleason alto y elevado PSA preoperatorio, fundamentalmente cuando hay márgenes positivos y el PSA previo a la radioterapia es inferior a $2 \mathrm{ng} / \mathrm{ml}$. Taylor $\mathrm{N}$ et al. en $2003^{69}$ evaluaron el papel de la radioterapia después de la prostatectomía radical en 2 grupos heterogéneos de pacientes. A un grupo de 75 pacientes con PSA postoperatorio indetectable, márgenes positivos en el $96 \%$ de los casos, le administraron una media de 60 Gy con carácter adyuvante. A otro grupo de 71 pacientes, 27 pacientes con PSA postoperatorio persistente- mente elevado, 44 con recidiva bioquímica de PSA, le administraron una media de 70 Gy con intención de "rescate". No sacaron conclusiones en relación con las dos modalidades comparadas de radioterapia. Kamat AM et al. en $2003^{70}$ intentaron identificar factores predictivos de respuesta a la radioterapia en pacientes con márgenes quirúrgicos positivos. De todos los parámetros analizados, el Gleason $\geq 7$ y el PSA preoperatorio mayor de $10.9 \mathrm{ng} / \mathrm{ml}$, eran los peores factores predictivos de recidiva bioquímica después de la radioterapia postoperatoria adyuvante. La radioterapia adyuvante es poco eficaz en pacientes con Gleason superior a 7 y PSA preoperatorio mayor de $10,9 \mathrm{ng} / \mathrm{ml}$, aunque el PSA previo a la radioterapia sea indetectable y los márgenes positivos. Valicenti RK et al. en $2004^{71}$ intentaron identificar un subgrupo de pacientes en tumores pT3N0M0, PSA preoperatorio indetectable y Gleason 7, con posibilidades de responder a la radioterapia adyuvante después de la prostatectomía radical. Los pacientes con márgenes quirúrgicos extensos en la pieza de prostatectomía son los que mejor parece que puedan beneficiarse de la radioterapia adyuvante. Sthephenson AJ et al. en $2004^{72}$.identificaron las variables predictoras más importantes de diseminación tumoral después de la prostatectomía radical: Gleason 8-10, PSA postoperatorio superior a $2 \mathrm{ng} / \mathrm{ml}$, Tiempo de Duplicación del PSA inferior a 10 meses e invasión de las vesículas seminales. $\mathrm{El}$ margen quirúrgico positivo es una variable que predice respuesta a la radioterapia de rescate después de la prostatectomía radical. El porcentaje de respuestas que obtuvieron con radioterapia de rescate en pacientes con márgenes positivos fue del $64 \%$ mientras que en los pacientes con márgenes negativos el porcentaje de respuestas fue del $22 \%$.

En nuestro trabajo, las características anatomopatológicas de los márgenes quirúrgicos positivos en las piezas de prostatectomía están implicados en la evolución posterior del cáncer. Por una parte, existe un primer grupo de pacientes con márgenes positivos con una morfología histopatológica que lleva implícito una alta probabilidad de riesgo de recidiva a los 5 años: riesgo de recidiva en márgenes positivos por extensión extraprostática $67 \%$, en márgenes espiculados 74\%, en márgenes de más de 4 mm 68\%, en már- 
genes multifocales $100 \%$. Por otra parte, existe un segundo grupo con márgenes positivos con una morfología anatomopatológica que conlleva un pronóstico más esperanzador: riesgo de recidiva en márgenes positivos por incisión capsular 26\%, en

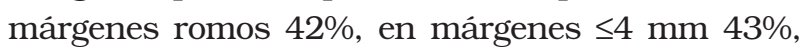
en márgenes únicos $47 \%$.

Según nuestra observación y las conclusiones de la literatura revisada anteriormente, una pauta de actuación en pacientes con márgenes positivos podría establecerse de la siguiente manera: Pacientes con márgenes positivos por extensión extraprostática, márgenes espiculados, márgenes de más de $4 \mathrm{~mm}$ o márgenes multifocales, con PSA preoperatorio menor de $10 \mathrm{ng} / \mathrm{ml}$ y Gleason $\leq 6$, tratamiento con radioterapia adyuvante; pacientes con márgenes positivos por extensión extraprostática, márgenes espiculados, márgenes de más de $4 \mathrm{~mm}$ o márgenes multifocales, con PSA preoperatorio mayor de $10 \mathrm{ng} / \mathrm{ml}$ o Gleason $\geq 7$, tratamiento hormonal; pacientes con márgenes positivos por incisión capsular, márgenes romos, márgenes $\leq$ 4 mm y márgenes únicos, observación y radioterapia de rescate o tratamiento hormonal, según la dinámica del PSA postoperatorio ${ }^{73}$. Si la dinámica postoperatoria del PSA sugiere recidiva local, radioterapia, y si sugiere recidiva sistémica, tratamiento hormonal.

\section{CONCLUSIONES}

De este trabajo se pueden extraer las siguientes conclusiones:

1. Que las características de los márgenes positivos están implicadas en la evolución del cáncer de próstata después de la prostatectomía radical.

2. Que se puede establecer un primer grupo de pacientes con márgenes positivos con una alta probabilidad de recidiva bioquímica: márgenes por extensión extraprostática, márgenes espiculados, márgenes de más de $4 \mathrm{~mm}$ y márgenes múltiples.

3. Que existe un segundo grupo de pacientes con márgenes positivos con un pronóstico más esperanzador en cuanto a la recidiva bioquímica: márgenes por incisión capsular, márgenes romos, márgenes $\leq 4 \mathrm{~mm}$ y márgenes únicos.

\section{REFERENCIAS}

1. Soloway MS, Wieder JA: Incidencia, etiologia, localización prevención y tratamiento de los bordes quirúrgicos positivos tras la prostatectomía por cáncer de próstata. En: Villavicencio H, Solsona E, editores. Estrategia actual para el futuro de la uro-oncología. Madrid. Acción Médica, 1997;87-126.

2. Epstein JI: Pathologic assessment of the surgical specimen. Urol Clin North Am 2001 Aug;28(3):567-594.

3. Epstein JI: The prostate and seminal vesicles. In: Carter D, Greenson JK, Oberman HA, Reuter VE, Stoler MH. Sternberg's diagnostic surgical pathology. Lippincott Philadelphia. Williams\&Wilkins, 2004, p.x-y. Vol 2.

4. Watson RB, Civantos F, Soloway MS. Positive surgical margins with radical prostatectomy: detailed pathological analysis and prognosis. Urology 1996 Jul;48(1):80-90.

5. Weldon VE, Tavel FR, Neuwirth H, Cohen R. Patterns of positive specimen margins and detectable prostate specific antigen after radical perineal prostatectomy. J Urol 1995 May; 153(5): 1565-1569.

6. Fesseha T, Sakr W, Grignon D, Banerjee M, Wood DP Jr, Pontes JE. Prognostic implications of a positive apical margin in radical prostatectomy specimens. J Urol 1997 Dec;158(6):2176-2179.

7. Cheng L, Slezak J, Bergstralh EJ, Myers RP, Zincke H, Bostwick DG. Preoperative prediction of surgical margin status in patients with prostate cancer treated by radical prostatectomy. J Clin Oncol 2000 Aug; 18(15):2862-2868.

8. De La Rosette JJ, Abbou CC, Rassweiler J, Laguna MP, Schulman CC. Laparoscopic radical prostatectomy: a European virus with global potentials. Arch Esp Urol 2002 Jul-Aug;55(6):603-609.

9. Berger AP, Volgger H, Rogatsch H, Strohmeyer D, Steiner H, Klocker $\mathrm{H}$ et al. Screening with low PSA cutoff values results in low rates of positive surgical margins in radical prostatectomy specimens. Prostate 2002 Nov 1;53(3):241-245.

10. Deliveliotis C, Protogerou V, Alargof E, Varkarakis J. Radical prostatectomy: bladder neck preservation and puboprostatic ligament sparing-effects on continence and positive margins. Urology 2002 Nov;60(5):855-858.

11. Salomon L, Anastasiadis AG, Levrel O, Katz R, Saint F, de la Taille A et al. Location of positive surgical margins after retropubic, perineal, and laparoscopic radical prostatectomy for organ-confined prostate cancer. Urology 2003 Feb;61(2):386-390.

12. Guillonneau B, el-Fettouh H, Baumert H, Cathelineau X, Doublet JD, Fromont G et al. Laparoscopic radical prostatectomy: oncological evaluation after 1.000 cases a Montsouris Institute. J Urol 2003 Apr;169(4):1261-1266.

13. Rassweiler J, Seemann O, Schulze M, Teber D, Hatzinger M, Frede T. Laparoscopic versus open radical prostatectomy: a comparative study at a single institution. J Urol 2003 May; 169(5):1689-1693.

14. Bianco FJ, Grignon DJ, Sakr WA, Shekarriz B, Upadhyay J, Dornelles E et al. Radical prostatectomy with bladder neck preservation: impact of a positive margin. Eur Urol 2003 May;43(5):461-466.

15. Cecchini Rosell L, Areal Calama J, Saladie Roig JM. Laparoscopic radical prostatectomy. Review of our first year. Arch Esp Urol 2003 Apr;56(3):287-293.

16. Khan MA, Han M, Partin AW, Epstein JI, Walsh PC. Longterm cancer control of radical prostatectomy in men younger than 50 years of age: update 2003. Urology 2003 Jul;62(1):86-91; discussion 91-92. 
17. Osorio Pazo F, Mouro Giudice L, Jubin Martínez J. Valor pronóstico en la prostatectomía radical del número de cilindros positivos y porcentaje de cáncer por cilindro de la biopsia prostática. Actas Urol Esp 2003 Jul-Ago;27(7):538542.

18. Brown JA, Garlitz C, Gomella LG, Hubosky SG, Diamond SM, McGinnis D et al. Pathologic comparison of laparoscopic versus open radical retropubic prostatectomy specimens. Urology 2003 Sep;62(3):481-486.

19. Laven BA, Alsikafi NF, Yang XJ, Brendler CB. Minor modifications in apical dissection of radical retropubic prostatectomy in patients with clinical stage T2 prostate cancer reduce positive surgical margin incidence. Urology 2004 Jan;63(1):95-98.

20. Serni S, Masieri L, Lapini A, Nesi G, Carini M. A low incidence of positive surgical margins in prostate cancer at high risk of extracapsular extension after a modified anterograde radical prostatectomy. BJU Int 2004 Feb;93(3): 279-83.

21. Varkarakis J, Pinggera GM, Sebe P, Berger A, Bartsch G, Horninger W. Radical retropubic prostatectomy in men younger than 45 years diagnosed during early prostate cancer detection program. Urology 2004 Feb;63(2):337-341.

22. Baumert H, Fromont G, Adorno Rosa J, Cahill D, Cathelineau X, Vallancien G. Impact of learning curve in laparoscopic radical prostatectomy on margin status: prospective study of first 100 procedures performed by one surgeon. J Endourol 2004 Mar;18(2):173-176.

23. Ruiz L, Salomon L, Hoznek A, Vordos D, Yiou R, de la Taille A et al. Comparison of early oncologic results of laparoscopic radical prostatectomy by extraperitoneal versus transperitoneal approach. Eur Urol 2004 Jul;46(1):50-54; discussion 54-56.

24. Martorana G, Manferrari F, Bertaccini A, Malizia M, Palmieri F, Severini E et al. Laparoscopic radical prostatectomy: oncological evaluation in the early phase of the learning curve comparing to retropubic approach. Arch Ital Urol Androl 2004 Mar;76(1):1-5.

25. Selli C, De Antoni P, Moro U, Macchiarella A, Giannarini G, Crisci A. Role of bladder neck preservation in urinary continence following radical retropubic prostatectomy. Scand J Urol Nephrol 2004;38(1):32-37.

26. Erdogru T, Teber D, Frede T, Marrero R, Hammady A, Seemann $\mathrm{O}$ et al. Comparison of transperitoneal and extraperitoneal laparoscopic radical prostatectomy using match-pair analysis. Eur Urol 2004 Sep;46(3):312-319.

27. Poulakis V, Witzsch U, de Vries R, Emmerlich V, Meves M, Altmannsberger $\mathrm{HM}$ et al. Preoperative neural network using combined magnetic resonance imaging variables, prostate-specific antigen, and gleason score for predicting prostate cancer biochemical recurrence after radical prostatectomy. Urology 2004 Dec;64(6):1165-1170.

28. Ward JF, Zincke H, Bergstralh EJ, Slezak JM, Myers RP Blute ML. The impact of surgical approach (nerve bundle preservation versus wide local excision) on surgical margins and biochemical recurrence following radical prostatectomy. J Urol 2004 Oct;172(4 Pt 1):1328-1332.

29. Gaker DL, Steel BL. Radical prostatectomy with preservation of urinary continence: pathology and long-term results. J Urol 2004 Dec;172(6 Pt 2):2549-2552.

30. Ackerman DA, Barry JM, Wicklund RA, Olson N, Lowe BA Analysis of risk factors associated with prostate cancer extension to the surgical margin and pelvic node metastasis at radical prostatectomy. J Urol 1993 Dec;150(6):1845-1850.
31. Stamey TA, Villers AA, McNeal JE, Link PC, Freiha FS. Positive surgical margins at radical prostatectomy: importance of the apical dissection. J Urol 1990 Jun;143(6):1166-1172.

32. Blute ML, Bostwick DG, Bergstralh EJ, Slezak JM, Martin SK, Amling CL et al. Anatomic site-specific positive margins in organ-confined prostate cancer and its impact on outcome after radical prostatectomy. Urology 1997 Nov;50(5):733-739.

33. Ohori M, Wheeler TM, Kattan MW, Goto Y, Scardino PT. Prognostic significance of positive surgical margins in radical prostatectomy specimens. J Urol 1995 Nov;154(5):1818-1824.

34. Watson RB, Civantos F, Soloway MS. Positive surgical margins with radical prostatectomy: detailed pathological analysis and prognosis. Urology $1996 \mathrm{Jul}$;48(1):80-90.

35. Ohori M, Abbas F, Wheeler TM, Kattan MW, Scardino PT, Lerner SP. Pathological features and prognostic significance of prostate cancer in the apical section determined by whole mount histology. J Urol 1999 Feb;161(2):500-504.

36. Cheng L, Darson MF, Bergstralh EJ, Slezak J, Myers RP, Bostwick DG. Correlation of margin status and extraprostatic extension with progression of prostate carcinoma. Cancer 1999 Nov 1;86(9):1775-1782.

37. Zudaire Bergera JJ, Martín-Marquina Aspiunza A, Sánchez Zalabardo D, Arocena García-Tapia J, Sanz Pérez G, Diez Caballero F, et al. Prostatectomía radical en adenocarcinoma de próstata clínicamente localizado. Estudio de los pacientes con márgenes positivos y su influencia en la supervivencia libre de progresión bioquímica. Actas Urol Esp 1999 Nov-Dec;23(10):835-842.

38. Kausik SJ, Blute ML, Sebo TJ, Leibovich BC, Bergstralh EJ, Slezak J et al. Prognostic significance of positive surgical margins in patients with extraprostatic carcinoma after radical prostatectomy. Cancer 2002 Sep 15;95(6): 1215-1219.

39. Salomon L, Anastasiadis AG, Antiphon P, Levrel O, Saint F, De La Taille A et al. Prognostic consequences of the location of positive surgical margins in organ-confined prostate cancer. Urol Int 2003;70(4):291-296.

40. Freedland SJ, Aronson W, Presti JC Jr, Kane CJ, Terris MK, Elashoff D et al. The SEARCH Database Study Group. Should a positive surgical margin following radical prostatectomy be pathological stage T2 or T3? Results from the SEARCH database. J Urol 2003 Jun;169(6):21422146.

41. Pettus JA, Weight CJ, Thompson CJ, Middleton RG, Stephenson RA. Biochemical failure in men following radical retropubic prostatectomy: impact of surgical margin status and location. Urol 2004 Jul;172(1):129-132.

42. Connolly SS, O’Toole GC, O'Malley KJ, Manecksha R, O'Brien A, Mulvin DW et al. Positive apical surgical margins after radical retropubic prostatectomy, truth or artefact? Scand J Urol Nephrol 2004;38(1):26-31.

43. Weldon VE, Tavel FR, Neuwirth H, Cohen R. Patterns of positive specimen margins and detectable prostate specific antigen after radical perineal prostatectomy. J Urol. 1995 May; 153(5): 1565-1569.

44. Fesseha T, Sakr W, Grignon D, Banerjee M, Wood DP Jr, Pontes JE. Prognostic implications of a positive apical margin in radical prostatectomy specimens. J Urol 1997 Dec; 158(6):2176-2179.

45. Shah O, Melamed J, Lepor H. Analysis of apical soft tissue margins during radical retropubic prostatectomy. J Urol 2001 Jun;165(6 Pt 1):1943-1948. 
46. Soulie M, Aziza R, Escourrou G, Seguin P, Tollon C, Molinier L et al. Assessment of the risk of positive surgical margins with pelvic phased-array magnetic resonance imaging in patients with clinically localized prostate cancer: a prospective study. Urology 2001 Aug;58(2):228-232.

47. Soloway MS, Pareek K, Sharifi R, Wajsman Z, McLeod D, Wood DP Jr et al. Lupron Depot Neoadjuvant Prostate Cancer Study Group. Neoadjuvant androgen ablation before radical prostatectomy in cT2bNxMo prostate cancer: 5year results. J Urol 2002 Jan;167(1):112-116.

48. Aus G, Abrahamsson PA, Ahlgren G, Hugosson J, Lundberg S, Schain M, et al. Three-month neoadjuvant hormonal therapy before radical prostatectomy: a 7-year follow-up of a randomized controlled trial. BJU Int 2002 Oct;90(6):561-566.

49. Sofer M, Hamilton-Nelson KL, Schlesselman JJ, Soloway MS. Risk of positive margins and biochemical recurrence in relation to nerve-sparing radical prostatectomy. J Clin Oncol 2002 Apr 1;20(7):1853-1858.

50. Goharderakhshan RZ, Sudilovsky D, Carroll LA, Grossfeld GD, Marn R, Carroll PR. Utility of intraoperative frozen section analysis of surgical margins in region of neurovascular bundles at radical prostatectomy. Urology. 2002 May;59(5):709-714.

51. Eastham JA, Kattan MW, Riedel E, Begg CB, Wheeler TM, Gerigk $\mathrm{C}$ et al. Variations among individual surgeons in the rate of positive surgical margins in radical prostatectomy specimens. J Urol 2003 Dec;170(6 Pt 1):2292-2295.

52. Shah O, Robbins DA, Melamed J, Lepor H. The New York University nerve sparing algorithm decreases the rate of positive surgical margins following radical retropubic prostatectomy. J Urol 2003 Jun;169(6):2147-2152.

53. Klotz LH, Goldenberg SL, Jewett MA, Fradet Y, Nam R, Barkin $\mathrm{J}$ et al. Canadian Uro-Oncology Group. Long-term followup of a randomized trial of 0 versus 3 months of neoadjuvant androgen ablation before radical prostatectomy. J Urol 2003 Sep;170(3):791-794.

54. Fromont G, Baumert H, Cathelineau X, Rozet F, Validire P, Vallancien $\mathrm{G}$. Intraoperative frozen section analysis during nerve sparing laparoscopic radical prostatectomy: feasibility study. J Urol 2003 Nov; 170(5):1843-1846.

55. Lepor H, Kaci L. Role of intraoperative biopsies during radical retropubic prostatectomy. Urology 2004 Mar;63(3): 499-502.

56. Prezioso D, Lotti T, Polito M, Montironi R. Neoadjuvant hormone treatment with leuprolide acetate depot $3.75 \mathrm{mg}$ and cyproterone acetate, before radical prostatectomy: a randomized study. Urol Int 2004;72(3):189-195.

57. Hricak H, Wang L, Wei DC, Coakley FV, Akin O, Reuter VE et al. The role of preoperative endorectal magnetic resonance imaging in the decision regarding whether to preserve or resect neurovascular bundles during radical retropubic prostatectomy. Cancer 2004 Jun; 100(12):26552663.

58. Ukimura O, Gill IS, Desai MM, Steinberg AP, Kilciler M, Ng $\mathrm{CS}$ et al. Real-time transrectal ultrasonography during laparoscopic radical prostatectomy. J Urol 2004 Jul; 172(1):112-118.

59. Han M, Partin AW, Chan DY, Walsh PC. An evaluation of the decreasing incidence of positive surgical margins in a large retropubic prostatectomy series. J Urol 2004 Jan;171(1):23-26.
60. Hennequin C, Ravery V, Maylin C, Boccon-Gibbod L. Postprostatectomy radiotherapy: for which patients and when? Cancer Radiother 2002 May;6(3):168-174.

61. Valicenti RK, Gomella LG, Pérez CA. Radiation therapy after radical prostatectomy: a review of the issues and options. Semin Radiat Oncol 2003 Apr;13(2):130-140.

62. Nelson JB, Lepor H. Prostate cancer: radical prostatectomy. Urol Clin North Am 2003 Nov;30(4):703-723.

63. Grossfeld GD, Tigrani VS, Nudell D, Roach M 3rd, Weinberg VK, Presti JC Jr et al. Management of a positive surgical margin after radical prostatectomy: decision analysis. J Urol 2000 Jul;164(1):93-99; discussion 100.

64. Han M, Pound CR, Potter SR, Partin AW, Epstein JI, Walsh PC. Isolated local recurrence is rare after radical prostatectomy in men with Gleason 7 prostate cancer and positive surgical margins: therapeutic implications. J Urol 2001 Mar;165(3):864-866.

65. Anscher MS. Adjuvant radiotherapy following radical prostatectomy is more effective and less toxic than salvage radiotherapy for a rising prostate specific antigen. Int $\mathrm{J}$ Cancer 2001 Apr 20;96(2):91-93.

66. Do LV, Do TM, Smith R, Parker RG. Postoperative radiotherapy for carcinoma of the prostate: impact on both local control and distant disease-free survival. Am J Clin Oncol 2002 Feb;25(1):1-8.

67. Hachiya T, Minei S, Hirano D, Ishida H, Okada K, Takimoto Y. Adjuvant hormone therapy in patients with positive surgical margins after radical prostatectomy. Nippon Hinyokika Gakkai Zasshi 2002 Mar;93(3):469-475.

68. Lennernas B, Edgren M, Haggman M, Norlen BJ, Nilsson S. Postoperative radiotherapy after prostatectomy-a review. Scand J Urol Nephrol 2003;37(1):10-15.

69. Taylor N, Kelly JF, Kuban DA, Babaian RJ, Pisters LL, Pollack A. Adjuvant and salvage radiotherapy after radical prostatectomy for prostate cancer. Int J Radiat Oncol Biol Phys 2003 Jul 1;56(3):755-763.

70. Kamat AM, Babaian K, Cheung MR, Naya Y, Huang SH, Kuban D et al Identification of factors predicting response to adjuvant radiation therapy in patients with positive margins after radical prostatectomy. J Urol 2003;Nov;170(5): 1860-1863.

71. Valicenti RK, Chervoneva I, Gomella LG. Importance of margin extent as a predictor of outcome after adjuvant radiotherapy for Gleason score 7 pT3NO prostate cancer. Int $\mathrm{J}$ Radiat Oncol Biol Phys 2004 Mar 15;58(4):1093-1097.

72. Stephenson AJ, Shariat SF, Zelefsky MJ, Kattan MW, Butler EB, Teh BS et al. Salvage radiotherapy for recurrent prostate cancer after radical prostatectomy. JAMA 2004 Mar17:291(11):1325-1332.

73. Ojea Calvo A, Pérez Rodríguez A, Domínguez Freire F, Alonso Rodrigo A, Rodríguez Iglesias B, Benavente Delgado $\mathrm{J}$ et al. Recidiva del cáncer de próstata después de la prostatectomía radical y radioterapia de rescate. Actas Urol Esp 2004;28(10):743-748.

\author{
Dr. A. Ojea Calvo \\ Complejo Universitario de Vigo \\ C/ Pizarro, 22 \\ 36204 Vigo \\ e-mail: dr.ojea@telefonica.net
}

(Trabajo recibido el 25 enero de 2005) 